\title{
Linx
}

Revue des linguistes de l'université Paris X Nanterre

$45 \mid 2001$

Invariants et variables dans les langues. Études typologiques

\section{Réflexions sur les catégories des unités linguistiques : comparaison de l'allemand et du chinois}

\section{André Rousseau}

\section{OpenEdition}

\section{Journals}

Édition électronique

URL : http://journals.openedition.org/linx/753

DOI : 10.4000/linx.753

ISSN : 2118-9692

\section{Éditeur}

Presses universitaires de Paris Nanterre

Édition imprimée

Date de publication : 1 décembre 2001

Pagination : $59-70$

ISSN : 0246-8743

\section{Référence électronique}

André Rousseau, «Réflexions sur les catégories des unités linguistiques : comparaison de l'allemand et du chinois », Linx [En ligne], 45 | 2001, mis en ligne le 19 juin 2012, consulté le 19 avril 2019. URL http://journals.openedition.org/linx/753; DOI : 10.4000/linx.753

Ce document a été généré automatiquement le 19 avril 2019

Département de Sciences du langage, Université Paris Ouest 


\section{Réflexions sur les catégories des unités linguistiques : comparaison de l'allemand et du chinois}

\section{André Rousseau}

1 La typologie, telle qu'elle est généralement pratiquée, consiste à confronter des langues de types différents à un moment donné de leur histoire et à chercher à dégager leurs convergences ou leurs propriétés spécifiques et éventuellement à mettre au jour des universaux.

2 Or, toutes les langues - sauf bien entendu les langues mortes - évoluent et sont en perpétuelle évolution. Ce facteur évolutif n'est en général pas pris en compte dans la typologie, qui à cet égard en est restée à l'opposition radicalisée par Saussure entre synchronie et diachronie.

3 Nous pensons qu'il est possible et souhaitable de réintégrer la diachronie dans la typologie, un peu à l'instar de la Phonologie panchronique de Cl. Hagège et A.G. Haudricourt, qui ont ainsi défini leur ouvrage comme "l'étude et la systématisation des types d'évolution dans l'histoire des langues les plus diverses» (1978: 11). Cet objectif ambitieux a de quoi décourager, d'autant plus que la morpho-syntaxe est infiniment plus délicate à manipuler que la phonologie. Néanmoins il nous semble quand même possible d'ouvrir quelques pistes et de commencer à poser quelques jalons.

4 La question centrale dont il sera débattu à l'occasion de cette comparaison entre l'allemand et le chinois est celle du devenir des catégories dans une typologie évolutive.

\section{Les données du problème}

5 Ces réflexions sur les catégories linguistiques se situent au carrefour de deux idées fondamentales, qui représentent les lignes de force de l'exposé :

- les différentes langues sont façonnées par les «catégories» (au sens de J. Fourquet, 1970), qui leur donnent la forme extérieure qu'appréhende le descripteur; 
- la typologie des langues a toujours été pratiquée de manière statique ; or il semble qu'elle soit en mesure d'éclairer des phénomènes d'évolution.

\subsection{La notion de « catégories"} actuellement dans deux sens qui peuvent paraitre assez proches, mais qui sont en fait le fruit de deux analyses foncièrement différentes. Si l'on n'y prend garde, une confusion fâcheuse peut s'installer dans les esprits, que certains dictionnaires de linguistique (par ex. Dubois et alii, 1973) continuent à entretenir sous les expressions de «catégories primaires » (i.e. lexicales) et de « catégories secondaires » (i.e. grammaticales).

On rassemble ainsi sous la même étiquette de catégories à la fois les "classes de mots" traditionnelles et les propriétés spécifiques qui produisent ces «classes de mots ", sans voir que ces propriétés sémantiques agissent sur des lexèmes nus pour en faire des « classes de mots ».

Peu de linguistes, à notre connaissance, se sont risqués à examiner dans une perspective généraliste les fondements d'une théorie des catégories. G. Galichet l'a fait dans le cadre du français : dans son Essai de grammaire psychologique $\left({ }^{1} 1947,{ }^{2} 1950\right)$, il consacre toute la seconde partie de son ouvrage (c'est-à-dire à peu près une cinquantaine de pages) à l'étude assez approfondie des "catégories grammaticales ", distinguant des catégories nominales (genre et nombre), des catégories verbales (temps, mode et voix), une catégorie de la caractérisation (le degré) et une catégorie de discours (la personne). Cette étude des catégories semble être chez Galichet l'approfondissement de la première partie consacrée aux espèces grammaticales. Mais comme cette première partie comprend aussi un chapitre sur les marques d'expèces (article et personne), on comprend que Galichet a eu du mal à reconnaître la spécificité des catégories et de leurs marques.

théorie de Jean Fourquet sur les groupes spécifiques (1970), caractérisés par des catégories sémantiques présente une originalité incontestable tout en manifestant une dette à l'égard d'une part de Galichet et d'autre part de Tesnière. Si l'on examine de près la théorie des groupes spécifiques, on s'aperçoit que seuls deux groupes possèdent vraiment des catégories spécifiques: le groupe nominal et le groupe verbal, qui correspond à l'énoncé verbal. Le groupe qualitatif ne possède pas de catégories, car le degré, qui donne naissance à des bases lexicales, ne peut prétendre au statut de catégorie. Les autres groupes sont essentiellement des groupes d'association (groupe prépositionnel et groupe subordonné).

Les catégories de Jean Fourquet s'inscrivent, à quelques exceptions près, dans la tradition grammaticale, mais leur conception est originale. Il est clair d'autre part que ces catégories, issues de la description de langues flexionnelles ou anciennement flexionnelles, ne sauraient être des catégories universelles, du moins sous cette forme, et qu'il faudra y apporter des retouches.

\subsection{Typologie et évolution}

11 Il faut distinguer dans les langues deux types d'évolution: les évolutions à court ou moyen terme, qui n'affectent pas en profondeur l'appartenance de la langue à tel ou tel type de langues et les évolutions à long terme qui, à travers différents cheminements, 
convergent avec d'autres mutations pour aboutir finalement à un changement de type de langues.

Pour traiter ce sujet de morpho-syntaxe et de sémantique catégorielle, la typologie présentée initialement par A.W. Schlegel, qui distinguait dans son introduction à ses Observations sur la langue et la littérature provençales (1818) les langues sans aucune structure grammaticale (entendant par là le chinois), les langues qui emploient des affixes et les langues à flexions, typologie reprise par W.von Humboldt (1836) et ensuite par A. Schleicher (1860), nous parait être assez bien adaptée. Cette typologie aboutit à distinguer essentiellement trois types de langues : les langues flexionnelles (par ex. les langues indo-européennes anciennes), les langues agglutinantes (comme le turc), et les langues isolantes (par ex. le chinois).

13 Les recherches contemporaines sur les témoignages anciens des langues indoeuropéennes, notamment celles menées par Fr. Bader (1973, 1983, etc.) ont montré l'origine entièrement agglutinante de certains mots indo-européens, par ex. lat. reciprocus < *re-co-pro-co-, qui était encore ressentie comme telle par Ennius (Rursus prorsus reciprocat fluctus feram Andromaque 104 « le flot ramène alternativement en avant et en arrière la bête »); et, de son côté, J. Haudry (1982: 20ss) a démontré de manière irréfutable l'origine postpositionnelle, c'est-à-dire agglutinative, des désinences de la flexion athématique et a traité la voyelle thématique comme un article postposé1. Les morphèmes de la flexion en indo-européen sont d'anciennes particules qui se sont soudées au lexème. A vrai dire, plusieurs signes de cette thèse se trouvaient déjà chez les grands comparatistes (cf. Max Müller ${ }^{2}$; K. Brugmann 1904 ; A. Meillet 1931 : 194ss ; etc.) ${ }^{3}$. On peut considérer aujourd'hui comme admis que l'indo-européen, langue flexionnelle par excellence, est passé par un stade antérieur où il était de type agglutinatif. De son côté, le chinois ancien possède des traces évidentes d'une flexion dérivationnelle (cf Sagart, 1999), comme l'avait déjà observé Jespersen.

Cela prouve en tout cas que les changements à long terme peuvent se traduire par une évolution typologique.

\subsection{Le rôle des catégories}

15 Arrivé à ce point, on peut se demander ce qui fait la différence entre les langues et surtout entre les types de langues. Ce ne sont certainement pas les unités lexicales, qui dans toutes les langues sont des lexèmes potentiellement polyvalents. Les mots des langues indo-européennes, une fois débarrassés de leurs marques ou morphèmes de catégories, présentent le même visage que les mots d'autres types de langues, y compris les langues isolantes. A cet égard, on peut comparer :

\begin{tabular}{|l|l|}
\hline (1a) chinois & gongzuo shijan « temps de travail » \\
\hline (1b) all. & Arbeit-s- zeit « temps de travail » \\
\hline (où all. -s- est purement un « glide » phonologique) \\
\hline
\end{tabular}



lexèmes indifférenciés en classe lexicale (nom ou verbe), mais pouvant donner naissance à l'un et à l'autre.

Les divergences entre les langues se rencontrent dans les spécificités syntaxiques et sémantiques, au titre desquelles il faut compter les relations (marquée par la position, les cas ou les prépositions) et surtout les catégories. Comparer les langues revient souvent à comparer les types de catégories, comme on a pu rapprocher les "classes nominales » dans les langues africaines du groupe Niger-Congo des «genres » dans les langues indoeuropéennes (Creissels 1995, 1999). Mais il faut aussi aller beaucoup plus loin dans cette voie.

18 En outre, si l'on rapproche - comme on l'a suggéré au début de cet exposé - typologie et évolution, il faut alors reconnaître dans les catégories le facteur décisif susceptible de créer les mutations. C'est précisément la voie que traçait $\mathrm{E}$. Benveniste il y a une quarantaine d'années déjà : «On peut alors imaginer une sorte de classification de ces catégories qui en montrerait l'agencement et les lois de transformation" (1966:6). Le programme dessiné par Benveniste est très ambitieux et nous sommes loin de pouvoir répondre à ces exigences. Néanmoins, il semble possible de tracer, à partir d'un exemple précis, les contours d'un tel édifice.

\section{Comparaison typologique entre l'allemand et le chinois}

19 Dans un cadre typologique, O. Jespersen (1922) avait insisté sur le rapprochement que l'on pouvait faire entre l'anglais et le chinois. Certes, l'allemand n'est pas parvenu au même stade d'évolution que l'anglais, notamment en ce qui concerne la morphologie casuelle, mais il présente néanmoins des traces d'évolution incontestables et convergentes et il y a un grand intérêt typologique, selon nous, à envisager cette confrontation inédite avec le chinois, langue bien décrite, qui permet en outre quelques incursions dans son histoire (A. Peyraube \& T. Wiebusch 1993 ; L. Sagart 1999). Après un bref rappel des raisons et conditions de cette comparaison, l'examen typologique abordera les deux domaines où existent une sémantique catégorielle : le domaine nominal et le domaine verbal.

\subsection{Les raisons et les conditions de cette comparaison}

Cette comparaison, qui peut paraître sinon saugrenue, du moins inattendue, a été précédée - comme nous venons de le rappeler - par les observations fort pertinentes de Jespersen qui avait eu l'intuition que l'anglais possédait déjà certaines caractéristiques d'une langue isolante.

21 L'allemand est certes encore loin d'être identique à l'anglais, cependant la perte ou l'abandon, même partiel, de marques pour une langue flexionnelle est un signe typologique qui ne trompe pas. Ainsi l'allemand présente deux signaux qui méritent intérêt et attention :

- certains substantifs dans des conditions précises d'emploi restent invariables :

\begin{tabular}{|l|l|l|l|}
\hline$(2)$ & drei & Stück & Zucker \\
\hline
\end{tabular}




\begin{tabular}{|l|l|l|l|}
\hline & Trois & morceau (invariable) & sucre \\
\hline & \multicolumn{2}{|l|}{ «trois morceaux de sucre » } \\
\hline
\end{tabular}

- la relation casuelle qui régit les rapports au sein du groupe nominal est absente :

\begin{tabular}{|l|l|l|l|}
\hline (3) & ein & Glas & \\
\hline & un & verre & \\
\hline & \multicolumn{3}{|l|}{ « un verre de bière ». } \\
\hline
\end{tabular}

Dans certains cas, notamment pour des noms dont le pluriel est inusité, l'usage de ce qu'il faut bien appeler un « classificateur » (Rousseau, 1995) est absolument devenu obligatoire en allemenand:

\begin{tabular}{|l|l|}
\hline (4) & ein Kopf Salat « une tête de salade » \\
\hline & zwei Kopf Rotkraut « deux têtes de chou rouge » \\
\hline & drei Kopf Blumenkohl « trois têtes de chou-fleur ${ }^{5}$ \\
\hline
\end{tabular}

Il est incontestable que l'allemand a acquis des propriétés d'un autre type de langues et qu'il devient dès lors possible de le comparer avec une langue comme le chinois.

\subsection{Le domaine nominal}

Dans le domaine nominal, le phénomène essentiel qui caractérise le chinois est naturellement la présence obligatoire d'un classificateur. Les principales caractéristiques du classificateur en chinois sont les suivantes ${ }^{6}$ :

- sa position est fixe : il est placé entre d'un côté le démonstratif et le numéral et de l'autre le substantif :

\begin{tabular}{|l|l|l|l|}
\hline (5) zhei & san & ben & shu \\
\hline DÉM. & trois & CL. & livre \\
\hline « ces trois livres " & & & \\
\hline
\end{tabular}

- sa fonction sémantique : après en avoir largement débattu, les auteurs concluent que « les CL. chinois expriment toujours une quantification [...] en revanche, ils ne qualifient pas toujours » $(1993: 57)$

Les spécialistes sont d'accord sur un point essentiel : au moyen du classificateur, on passe d'une référence générique ou collective à une référence individualisée. C'est l'opposition entre type et occurrence. 

" classes nominales » reste très complexe, même si certains auteurs cherchent à apporter des distinctions nécessaires ${ }^{7}$. Ainsi dans le schéma proposé par C. Grinewald (1999 : 110), les classificateurs sont bien distincts à la fois des «noms de mesure» et des «classes nominales" ou genres, mais ils se décomposent eux-mêmes en "classificateurs mensuratifs » et en "classificateurs de tri ». Cette dualité semble bien être inhérente à tout classificateur : s'il quantifie, il qualifie en même temps (un verre d'eau) et inversement s'il qualifie, il est amené à quantifier (une rangée d'arbres).

L'allemand a développé plusieurs types de classificateurs que nous avons présentés dans un article antérieur (Rousseau, 1995a). Ils sont identifiables car ils sont grammaticalisés comme tels dans la mesure où ils se caractérisent par l'introduction de propriétés nouvelles, contraires à l'usage de l'allemand :

\begin{tabular}{|l|l|}
\hline (6) ein Glas Wasser & « un verre d'eau » \\
\hline \hline ein Satz Schüsseln & « un jeu de plats » \\
\hline
\end{tabular}

Dans tous ces exemples, le classificateur est invariable au pluriel et la relation classificateur-nom reste non-marquée (ce qui a conduit quelques grammairiens à parler d'« apposition »!).

Toutefois, certains d'entre eux sont seulement en voie ou en cours de grammaticalisation et ils conservent certaines propriétés des noms :

(7) ein Wald von Schornsteinen « une forêt de cheminées ».

30 Comme le montrent ces exemples, les classificateurs de l'allemand proviennent tous de substantifs, comme c'est aussi le cas en chinois (Peyraube \& Wiebusch pp. 57ss) et la langue allemande fournit trois filières propices à la genèse d'une expression nominale comportant un classificateur :

31 1) l'abandon pur et simple de toute marque de relation (principalement au génitif) :

(8) ein Glas roten Weines (gén.) > ein Glas roter Wein (nom.)

« un verre de vin rouge »

En l'occurrence, seul roter Wein est fléchi suivant le cas voulu par sa fonction dans l'énoncé :

(9) ich möchte ein Glas roten Wein (acc.)

« je voudrais un verre de vin rouge ».

2) l'éclatement d'un mot composé, qui implique un changement d'ordre (qui s'est produit également en chinois) :

\begin{tabular}{|l|l|l|}
\hline$(10)$ ein Blumenstrauß & $>$ & ein Strauß Blumen \\
\hline « un bouquet de fleurs » & & «id. » \\
\hline
\end{tabular}

3) le renversement d'une construction en als (Rousseau, 1995b) : 


\begin{tabular}{|l|l|l|}
\hline (11) Paris als Stadt & $>$ & die Stadt Paris \\
\hline \hline «Paris comme ville » & & « la ville de Paris » \\
\hline
\end{tabular}

Ces modèles d'obtention d'un classificateur donnent une idée de la variété des types de classificateurs dont dispose l'allemand.

Enfin, de même que le chinois présente un classificateur universel en ge:

\begin{tabular}{|l|l|l|l|l|l|l|}
\hline (12) shan & ge & shu & au lieu de : & shan & ben & shu \\
\hline trois & CL. UNIV. & livre & & & trois & CL \\
\hline livre & & & & & & \\
\hline \multicolumn{2}{|l|}{ «trois livres " } \\
\hline
\end{tabular}

de même l'allemand dispose lui aussi d'un classificateur général :
\begin{tabular}{|l|l|l|}
\hline (13) eine & Art & Kuchen \\
\hline
\end{tabular}
une sorte de gâteau »

\subsection{Le domaine verbal}

La comparaison typologique portera d'abord sur les catégories; mais il faudra évoquer également la généralisation des auxiliaires en allemand et le comportement très proche $\mathrm{du}$ « complexe prédicatif » en chinois et en allemand.

\subsubsection{Les catégories verbales.}

Le chinois connaît deux types de catégories verbales :

- des oppositions « aspectuelles ", marquées par des particules, entre deux types de procès :

(14)

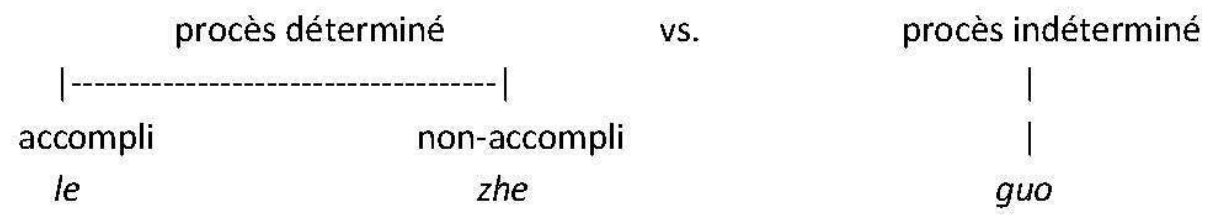

- une opposition au sein de l'Aktionsart « mode de procès », marquée aussi par des particules :

\begin{tabular}{|l|l|l|} 
(15) état & vs. & entrée dans l'état (ingressif) \\
\hline
\end{tabular} 


\begin{tabular}{|l|l|l|}
\hline zhe & & qi(lai); xia(lai). \\
\hline
\end{tabular}

L'allemand, de son côté, connaît les oppositions aspectuelles (accompli vs. non-accompli) et les oppositions d'Aktionsart, où la langue a recours à des auxiliaires, appelés « verbes fonctionnels » :

\begin{tabular}{|l|l|l|}
\hline (16) état & vs & ingressif \\
\hline in Kraft sein & & in Kraft treten \\
\hline Ansgt haben & & Angst bekommen \\
\hline
\end{tabular}

41 Mais l'allemand conserve par ailleurs des oppositions de temps et de modes, marquées essentiellement par des morphèmes verbaux et que ne semble pas connaître le chinois.

\subsubsection{La généralisation des verbes auxiliaires en allemand}

42 L'allemand manifeste une tendance très forte à utiliser des auxiliaires, dont nous essayons de donner un inventaire rapide :

- l'accompli est marqué par les auxiliaires sein et haben (+part. II) et cet accompli remplace même le prétérit en oberdeutsch ;

- le futur est rendu par l'auxiliaire werden + infinitif et le passif est exprimé par les auxiliaires sein et werden + participe II passif (cf Rousseau, 2000) ;

- le subjonctif II à valeur d'irréel fait très souvent appel aux auxiliaires würde ou täte + infinitif ;

- les « verbes fonctionnels » sont eux aussi des auxiliaires au service de l'Aktionsart ;

- enfin, la tournure tun + infinitif, employée en allemand standard seulement pour mettre en relief l'infinitif, est généralisée dans les dialectes : er tut schreiben « il écrit ».

43 Cette dichotomie verbale (auxiliaire + verbe plein) favorise apparemment le rendement syntaxique de l'assertion et de l'interrogation : l'auxiliaire seul occupe alors la première ou la seconde position et le verbe plein est placé à la finale, qui est sa position dans l'ordre structural. Mais en même temps, cette bipartition fonctionnelle du prédicat verbal a un effet pervers et lourd de conséquences : elle met en place une véritable stratégie de contournement et donc d'élimination de la morphologie verbale.

Il s'agit là d'un stade transitoire dans lequel fléchir seulement l'auxiliaire permet de conjuguer tous les verbes. Il peut être le prélude à un second stade où l'auxiliaire cessera à son tour d'être fléchi.

\subsubsection{Le comportement du « complexe prédicatif »}

C'est certainement le secteur où les deux langues, allemand et chinois, présentent le plus d'affinités dans le domaine verbal. En effet, l'expression du mouvement et de la direction offre des parallélismes étonnants entre les deux langues. 
La proximité se révèle d'abord au niveau des matériaux utilisés. Le chinois distingue trois séries de verbes :

1. une liste ouverte de verbes de mouvement : zao " partir ", pao « courir ", tiao « sauter ", dan " porter », na « prendre », etc.

2. une série restreinte de sept verbes de direction : shang " monter» vs. xia « descendre »; jin « entrer » vs. chu « sortir»; hui « retourner »; guo « traverser »; qi « se lever».

3. enfin un couple de verbes déictiques indiquant l'éloignement ou le rapprochement par rapport au locuteur : lai « venir » vs. qu « aller».

L'allemand répond par trois séries pratiquement identiques :

1. un inventaire ouvert de verbes de mouvement : laufen " courir ", springen "sauter ", schreiten « marcher ", steigen « faire un mouvement vertical », nehmen « prendre », tragen " porter », etc.

2. un inventaire limité de préverbes de direction: auf «monter » vs. ab «descendre »; ein «entrer» vs. aus «sortir»; über «par-dessus» vs. unter «par dessous»; durch «traverser »; um « renverser »; etc.

3. là aussi il existe un couple de préverbes déictiques orientés par rapport au locuteur : her « rapprochement » vs. hin « éloignement ».

En examinant le fonctionnement de ces trois éléments prédicatifs, il apparaît nettement que le chinois hésite entre la coverbation et le déplacement de (pré)verbes (Alleton, 1973 : 83-87), alors que l'allemand a résolument choisi de développer la syntaxe des préverbes (Rousseau, 1995c). Il y a donc entre les deux langues des identités remarquables de constructions, mais aussi des irréductibilités. Commençons par les parrallélismes :

\begin{tabular}{|c|c|c|c|c|c|c|c|}
\hline (17a) & ta & na & yi - ben & \multicolumn{2}{|l|}{ shu } & \multicolumn{2}{|l|}{ lai } \\
\hline & il & prendre & un CL. & \multicolumn{2}{|l|}{ livre } & \multicolumn{2}{|c|}{ venir } \\
\hline & \multicolumn{7}{|c|}{ «il apporte un livre » } \\
\hline (17b) & er & bringt & & ein & Buch & & her(an) \\
\hline & il & apporter-3-SG & & un & livre & & vers ici \\
\hline \multirow[t]{3}{*}{$(18 a)$} & ta & na & chu & yi - ben & shu & & lai \\
\hline & il & prendre & sortir & un - CL & livre & & venir \\
\hline & \multicolumn{6}{|c|}{ « il apporte un livre» } & \\
\hline$(18 b)$ & er & bringt & & ein & Buch & & Her - aus \\
\hline & il & apporte-3-SG & & un & livre & & ici - sortir \\
\hline (19a) & ta & na & yi - ben & shu & chu & & lai \\
\hline & il & prendre & un CL. & livre & sortir & & venir \\
\hline (19b) & er & bringt & ein Buch & her-aus & $(=18 b)$ & & \\
\hline
\end{tabular}


Le chinois semble avoir à sa disposition soit la coverbation (ex. (18a)), soit la copréverbation (ex. (19a)). Les mêmes énoncés du chinois peuvent donner lieu à des coverbations inconnues de l'allemand. Sauf à faire intervenir l'étymologie: on sait en effet, depuis la démonstration de Brugmann (1901), que l'allemand bringen « apporter » est en fait issu de l'agglutination d'une ancienne coverbation en indo-européen (*bher"porter» + $(\mathrm{e})$ nek "atteindre »). Dès lors, il est clair que l'allemand et plus anciennement le germanique ont possédé les mêmes possibilités d'association sémantique que celles rencontrées actuellement en chinois. Ce n'est pas un hasard si nous retrouvons une parité entre évolution des langues et typologie.

\section{III) L'évolutions des catégories}

50 La langue allemande est bien engagée dans un processus d'évolution à longue échéance qui concerne son passage de langue synthétique à langue analytique, comme l'est par exemple le chinois.

51 Nous avons travaillé avec l'hypothèse forte que les langues évoluent et surtout que cette évolution à long terme aboutit en fin de compte à une mutation des catégories. Or il semble bien qu'il y ait un net décalage entre l'évolution des catégories verbales et celle des catégories nominales.

\subsection{L'évolution des catégories verbales}

52 L'évolution des catégories verbales est incontestablement freinée en allemand. Il est possible d'en découvrir les causes dans l'examen approfondi, comme nous l'avons fait (Rousseau, 1995c), du fonctionnement du « complexe prédicatif ». Le diagnostic est assez facile à établir. Le verbe allemand est partie prenante dans deux stratégies qui, si elles visent à long terme la même finalité, se contrarient néanmoins fortement sur le terrain :

- d'une part, le développement des auxiliaires ;

- d'autres part, l'essor des préverbes.

53 Tiraillé entre ces deux actions antinomiques, le verbe allemand est frappé de paralysie ou d'immobilisme (relatif) en ce qui concerne l'évolution des catégories verbales.

54 Et pourtant, à regarder l'allemand de près, on constate qu'il dispose déjà d'un système cohérent de particules de temps (früher, jetzt, später, einst, etc.) et d'un ensemble homogène de particules aspectuelles (schon, noch,noch nicht, nicht mehr); mais il conserve intactes dans leur marquage ses catégories verbales spécifiques, notamment le temps et le mode. En revanche, la situation des catégories nominales est très différente.

\subsection{L'évolution des catégories nominales}

55 L'évolution qui se dessine à propos des catégories nominales nous permet de saisir les conditions favorables à la mutation des catégories. Trois facteurs principaux activent le renouvellement des catégories. 


\subsubsection{L'usure des catégories.}

56 Ce phénomène est particulièrement net pour la catégorie du nombre, qui était initialement beaucoup plus développée que ne le laisse penser l'actuelle opposition très réductrice entre un singulier et un pluriel. Cette catégorie comportait au moins deux séries de marques :

- pour le non-dénombrable ou massif, on pouvait opposer un singulatif à un collectif (comme en all. mod. Wasser vs. Gewässer) et il y avait aussi un paucal (all. ein weinig, ein bißchen);

- pour le dénombrable, on pouvait distinguer entre singulier, duel, éventuellement triel et quatriel (encore attestés dans les langues mélanésiennes et de Nouvelle-Calédonie), et pluriel.

\subsubsection{La perte de pertinence des catégories}

57 Ce trait affecte surtout la catégorie du genre. IL est très vraisemblable que l'indoeuropéen ancien ne disposait que d'une opposition binaire entre deux genres (animé vs. non-animé), divisant ensuite l'animé en masculin et féminin pour aboutir aux trois genres bien connus (Rousseau, 2001).

Mais dans une langue comme l'allemand moderne, le genre a perdu en grande partie sa pertinence référentielle: en effet, il ne fonctionne de manière satisfaisante que pour une partie des animés (êtres humains et grands animaux) :

(20a) der Mann / die Frau / das Kind « l'homme, la femme, l'enfant » der Stier / die Kuh / das Kalb « le taureau, la vache, le veau »;

pour le reste (petits animaux et objets), il est devenu une sorte de servitude, dénuée de toute valeur référentielle :

(20b) der Löffel / die Gabel / das Messer « la cuillère, la fourchette, le couteau »

60 Cette trilogie n'a rien de fonctionnel du point de vue linguistique ; elle n'est que le fruit du hasard. Cette situation favorise évidemment sa disparition et son remplacement.

\subsubsection{L'imbrication des catégories.}

61 Un autre critère hâtant la mise en place de nouvelles catégories concerne leur imbrication. La principale imbrication touche les catégories déjà évoquées de genre et de nombre : l'allemand moderne présente une imbrication curieuse concernant le genre et le nombre, qui se traduit par un amalgame de leurs signifiants respectifs et surtout une répartition nouvelle - phénomène sur lequel les grammaires sont pratiquement muettes.

- l'allemand moderne possède bien trois genres, mais au singulier seulement et n'a aucune marque de nombre au singulier, sinon $\varnothing$.

- l'allemand n'a aucune marque de genre au pluriel; en revanche il y a plusieurs morphèmes de pluriel.

Il existe bien une véritable répartition des marques: les marques de genre ne sont présentes qu'au singulier ; les marques de nombre n'apparaissent qu'au pluriel.

63 Cette imbrication entre genre et nombre et par conséquent leur perte de pouvoir distinctif est un facteur décisif pour l'apparition des classificateurs, qui possèdent comme nous l'avons indiqué - la double fonction : à la fois mensuratifs et de tri. 
64 les catégories. Cette étude des catégories sémantiques, dans laquelle nous avons cherché à relier typologie et évolution des langues, a permis d'examiner les conditions de mutation des catégories en allemand à partir de l'éclairage apporté par le chinois. La conclusion apparaît sans ambiguïé : genres et nombre ayant perdu une grande partie de leur pertinence référentielle, la langue a recours à d'autes types de catégories, comme les classificateurs. Mais ce processus n'en est qu'à sa phase de préparation : à la différence du chinois, l'emploi des classificateurs en allemand n'est encore nullement obligatoire - à l'exception de quelques cas : zwei Kopf Salat, drei Kopf Kohl.

67 A côté de sa morphologie de langue flexionnelle, encore riche et variée, mais en recul au fil des siècles, l'allemand a commencé à mettre en place un dispositif comparable à celui du chinois, qui n'est pas encore opérationnel. Ce mouvement a débuté dès le stade du germanique quand les les cas ont été relayés par des prépopositions (Rousseau, 2001b), rendant ainsi souvent redondant l'usage des cas après une préposition. Il reste, on le voit, beaucoup de chemin à parcourir car ces évolutions typologiques se déroulent sur le long terme, étalées sur des siècles, voire des millénaires.

\section{BIBLIOGRAPHIE}

ALLETON, Viviane (1973) Grammaire du chinois. (Que sais-je n 1519). Paris, PUF.

BADER, Françoise (1973) « Lat. nempe, porceo et les fonctions des particules pronominales ». in :

BSLP 68. pp. 27-75.

BADER, Françoise (1983) « Fonctions et étymologies pronominales ». in : L'Information grammaticale

17. pp. 5-9 \& 18. pp. 9-13.

BENVEnISTE, Emile (1966) Problèmes de linguistique générale. tome I. Paris, Tel.

BRUGMANN, Karl (1901) « Homerisch menoinao und gotisch briggan, zwei Fälle von

Wurzelangleichung ». in IF 12. pp. 150-158.

BRUGMANN, Karl (1904) Kurze vergleichende Grammatik der indogermanische Sprachen.

Strasbourg, Trübner.

Linx, 45 | 2001 
CREISSELS, Denis (1995) Eléments de synyaxe générale. Paris, PUF.

CREISSELS, Denis (1999) «' 'Genres' indo-européens et 'classes nominales' Niger-Congo ». in Faits de langues 14. pp. 177-184.

FOURQUET, Jean (1970) Prolegomena zu einer deutschen Grammatik. Düsseldorf, Schwann.

GALICHET, Georges (1950) Essai de grammaire psychologique. Paris, PUF.

GRINEWALD, Colette (1999) « Typologie de classification nominale ». in : Faits de langues 14.

pp. 101-122.

HAGÈGE, Claude (41995) La structure des langues. (Que sais-je n² 2006). Paris, PUF.

HAGEGE, Cl. \& HAUDRICOURT, a. (1978) La phonologie panchronique. Paris, PUF.

HAUDRY, jean (1982) Préhistoire de la flexion nominale indo-européenne. Lyon, Université Jean-

Moulin

Jespersen, Otto (1922) Nature, évolution et origine du langage. (trad. fr. 1976) Paris, Payot.

LEMARECHAL, Alain (1997) « Superposition des marques, morphologisation et zéro ». in : MSL V.

pp. 25-61.

LI, C.N. \& THompson S. A. (1993) Mandarin Chinese. A Functional Reference Grammar. Berkeley, University of California Press.

MEILLET, Antoine (1931) « Caractère secondaire du type thématique indo-européen ». in : BSLP 31. pp. 194-203.

PEYRAube, A. \& Wiebusch, Th. (1993) «Le rôle des classificateurs nominaux en chinois et leur évolution historique : un cas de changement cyclique ». in Faits de Langues 2. pp. 51-61.

ROUSSEAU, André (1995a) « Ist das gegenwärtige Deutsch eine 'Klassensprache' ? » in : Signans und Signatum. (Faucher, Métrich \& Vuillaume éd.) Tübingen, Narr. pp. 21-38

ROUSSEAU, André (1995b) «Als und wie : semantische Operatoren in den Nominalgruppen. Ein Beitrag zur natürlichen Logik ». in Rand und Band. (Métrich \& Vuillaume éd.) Tübingen, Narr. pp. 315-335.

ROUSSEAU, André éd. (1995c) Les Préverbes dans les langues d'Europe. Lille, Septentrion.

ROUSSEAU, André (2000) «Les 'opérateurs de prédication' dans les langues naturelles et leur grammaticalisation ». in Grammaticalisation I (Travaux linguistiques du CERLICO 13) pp. 31-57.

ROUSSEAU, André (2001a) «L'émergence du féminin en indo-européen » in : Ameida \& Maillard éd. O Feminino nas Linguas, Culturas e Litaratures. Madère, METAGRAM. pp. 375-383.

ROUSSEAU, André (2001b) «La sémantique des relations casuelles » in : A. Rousseau éd. La sémantique des relations. Lille, CGES pp. 49-70.

SAGART, Laurent (1999) The Roots of Old Chinese. Amsterdam, John Benjamins.

\section{NOTES}

1. J'invite les sceptiques à prendre connaissance des ouvrages et articles cités en référence, car ils font autorité.

2. Max Müller (1823-1990), Allemand de naissance, professeur à Oxford en 1850, écrit dans La sratification du langage: "partout où la flexion a cédé à une analyse rationnelle, elle a été 
invariablement reconnue pour le résultat d'une agglutination antérieure » (cité d'après Haudry $1982: 64)$

3. pour ne pas parler de Carl Meinhof (1936) Die Entstehung der flektierenden Sprachen. Berlin, ni de Franz Specht (1944) Der Ursprung der Indogermanischen Dekination. Göttingen, Vandenhoeck \& Ruprecht.

4. alors que le mot Stück a bien un pluriel en Stücke.

5. là encore, Kopf a un pluriel habituel en Köpfe.

6. nous nous appuyons essentiellement sur l'excellent article de Peyraube et Wiebusch publié dans Faits de langue 2 (1993 : 51-61).

7. C'est le cas de l'article très clair de Colette Grinewald dans le $\mathrm{n}^{\circ} 14$ de Faits de langues.

\section{AUTEUR}

\section{ANDRÉ ROUSSEAU}

Univeristé Charles de Gaulle - Lille III E.A. SELOEN 2455 